\title{
About the Images and Inverse Images Of Intuitionistic or Vague Fuzzy Subsets
}

\author{
G. Vasanti \\ Associate Professor, Department of Basic Sciences and Humanities \\ Aditya Institute of Technology and Management, Tekkali.
}

Keywords: Intuitionistic Fuzzy or Vague Subset, Intuitionistic Fuzzy (Inverse) Image.

Abstract. In this paper an exclusive study of some standard (lattice) algebraic properties for Intuitionistic fuzzy (inverse) images of Intuitionistic fuzzy subsets is done. Further as in crisp setup, characterizations for injectivity and surjectivity of maps in terms of some (lattice) algebraic properties of Intuitionistic fuzzy images and Intuitionistic fuzzy inverse images are performed.

\section{Introduction}

The traditional view in science, especially in mathematics, is to avoid uncertainty at all levels at any cost. Thus, 'being uncertain' is regarded as 'being unscientific'. But unfortunately in real life most of the information that we have to deal with is mostly uncertain.

One of the paradigm shifts in science and mathematics in this century is to accept uncertainty as part of science and the desire to be able to deal with it, as there is very little left out in the practical real world for scientific and mathematical processing without this acceptance!

One of the earliest successful attempts in this direction is the development of the Theories of Probability and Statistics.

However, both of them have their own natural limitations. Another successful attempt again in the same direction is the so called Fuzzy Set Theory, introduced by Lotfi Zadeh.

According to Zadeh, a fuzzy Subset of a set $X$ is a function $\mu$ from $X$ to the closed interval $[0,1]$ of real numbers. The function $\mu$, he called, the membership function which assigns to each member $\mathrm{X}$ of $X$ its membership value, $\mu \mathrm{x}$ in $[0,1]$.

Fuzzy set theory is one area with large number of applications both in Mathematics and in Computer science. For applications of fuzzy set theory in Mathematics, one can refer to the text books Mordeson and Malik[10] in Fuzzy Algebra and Ying-Ming and Mao-Kang[15] in Fuzzy Topology. For applications of Fuzzy set theory in Computer science, one can refer to the text books Galindo-Urrutia and Piattini[5] in Fuzzy Data Base Management Systems, Tamalika and Ajoy[12] in Fuzzy Image Processing, Hiroshi[7] and Morgan[9] in Fuzzy Data Mining, Zhang[14] and Liu and Li[11] in Fuzzy Neural Networks, Valente and Pedrycz[8] in fuzzy clustering.

In 1983, Atanassov[1] generalized the notion of Zadeh fuzzy subset of a set further by introducing an additional function $v$, which he called a non-membership function with some natural conditions on $\mu$ and $v$, calling these new generalized fuzzy subsets of a set, Intuitionistic fuzzy subsets. Thus according to him an Intuitionistic fuzzy subset of a set $X$, is a pair $A=(\mu \mathrm{A}, v \mathrm{~A})$, where $\mu \mathrm{A}, v \mathrm{~A}$ : $X \rightarrow[0,1]$ of real numbers such that for each $x$ in $X, \mu(x)+v(x) \leq 1$, where $\mu A$ is called the membership function of $\mathrm{A}$ and $\mu \mathrm{A}$ is called the non -membership function of $\mathrm{A}$.

Interestingly the same notion of Intuitionistic fuzzy subset of a set was also introduced by Gau and Buehrer[6] in 1993 under a different name called Vague subset. Thus whether we called Intuitionistic fuzzy subset of a set or if-subset of a set for short, or vague subset of a set, they are one and the same.

In stead of using long phrases like Intuitionistic fuzzy subset or vague subset, here onwards, we use the phrase if-subset. Obviously, if/v-subset only means Intuitionistic fuzzy/vague subset. 
Ever since Atanassov[1] introduced the notion of Intuitionistic fuzzy subset of a set, several mathematicians started imposing and studying both algebraic and topological structures on Intuitionistic fuzzy subsets. Looking at several of the papers that are in print and online, one thing which becomes evident is that various (lattice) algebraic properties of images and inverse images of a Intuitionistic fuzzy subset which, incidentally, not only play a crucial role in the study of both Intuitionistic Fuzzy Algebra and Intuitionistic Fuzzy Topology but also are necessary for the individual/ exclusive development of Intuitionistic Fuzzy Set Theory, are not yet studied, although these concepts were existing since long. In fact, these concepts of if $/ v$-image and if $/ \mathrm{v}$ inverse image are dealt in Ming[17], Thakur and Pandey[13], Davvaz, Dudek and Jun[4], Yon, Jun and Kim[16]. However, in this paper, we make an exclusive and somewhat detailed study of these (lattice) algebraic properties of Intuitionistic fuzzy images and Intuitionistic fuzzy inverse images under crisp maps. Further as in crisp setup, we characterize some injectivity and surjectivity of maps in terms of some (lattice) algebraic properties of Intuitionistic fuzzy images and Intuitionistic fuzzy inverse images.

A few of the results in this paper may be available in the literature elsewhere but scattered; however, we presented them here not only collectively but also in a suitable way for further research to the individual/exclusive development of Intuitionistic fuzzy Set Theory.

For any set $\mathrm{X}$, the set of all if-subsets of $\mathrm{X}$ be denoted by $\mathrm{I}(\mathrm{X})$. By defining, for any pair of if-subsets $A=\left(\mu_{A}, v_{A}\right)$ and $B=\left(\mu_{B}, v_{B}\right)$ of $X, A \leq B$ iff $\mu_{A} \leq \mu_{B}$ and $v_{B} \leq v_{A}, I(X)$ becomes a complete infinitely distributive lattice. In this case for any family $\left(\mathrm{A}_{\mathrm{i}}\right)_{\mathrm{i} \in \mathrm{I}}$ of ifsubsets of $\mathrm{X}, \mathrm{V}_{\mathrm{i} \in \mathrm{I}} \mathrm{Ai}=\left(\mathrm{V}_{\mathrm{i} \in \mathrm{I}} \mu_{\mathrm{Ai}}, \Lambda_{\mathrm{i} \in \mathrm{I}} \mathrm{v}_{\mathrm{Ai}}\right)$ and $\Lambda_{\mathrm{i} \in \mathrm{I}} \mathrm{Ai}=\left(\Lambda_{\mathrm{i} \in \mathrm{I}} \mu_{\mathrm{Ai}}, \mathrm{V}_{\mathrm{i} \in \mathrm{I}} \mathrm{v}_{\mathrm{Ai}}\right)$, where for $\lambda_{\mathrm{i}}: \mathrm{X} \rightarrow[0,1],\left(\mathrm{V}_{\mathrm{i} \in \mathrm{I}} \lambda_{\mathrm{i}}\right) \mathrm{x}=\mathrm{V}_{\mathrm{i} \in \mathrm{I}} \lambda_{\mathrm{i}} \mathrm{x}$ and $\left(\Lambda_{\mathrm{i} \in \mathrm{I}} \lambda_{\mathrm{i}}\right) \mathrm{x}=\Lambda_{\mathrm{i} \in \mathrm{I}} \lambda_{\mathrm{i}} \mathrm{x}$.

For any set $X$, one can naturally associate, with $X$, the if-subset $\left(\mu_{X}, v_{X}\right)=\left(1_{X}, 0_{X}\right)$, where $l_{X}$ is the constant map assuming the value 1 for each $\mathrm{x} \in \mathrm{X}$ and $0_{\mathrm{X}}$ is the constant map assuming the value 0 of for each $\mathrm{x} \in \mathrm{X}$, which turns out to be the largest element in $\mathrm{I}(\mathrm{X})$. Observe that then, the if-empty subset $\Phi$ of $X$ gets naturally associated with the ifsubset $\left(\mu_{\Phi}, v_{\Phi}\right)=(0,1)$, which turns out to be the least element in $\mathrm{I}(\mathrm{X})$.

Let $A=\left(\mu_{A}, v_{A}\right)$ be an if-subset of $X$. Then it turns out that $\left(v_{A}, \mu_{A}\right)$ is also an if-subset of $X$, thus for any if-subset $A=\left(\mu_{A}, v_{A}\right)$ the if-complement of $A$, denoted by $A^{c}$ is defined by $\left(v_{A}, \mu_{A}\right)$. Observe that $A^{c}=X-A=X \Lambda A^{c}$. Further for any pair $A, B$ of if $/ v$-subsets of $X$, we define $B-A$ to be $B \Lambda A^{c}$. In other words, for if $/ v$-subsets $B=\left(\mu_{B}, v_{B}\right)$ and $A=$ $\left(\mu_{\mathrm{A}}, v_{\mathrm{A}}\right)$ of $\mathrm{X}, \mathrm{B}-\mathrm{A}=\left(\mu_{\mathrm{B}} \Lambda \mathrm{v}_{\mathrm{A}}, \mathrm{v}_{\mathrm{B}} \Lambda \mu_{\mathrm{A}}\right)$.

Throughout this paper the capital letters $X, Y, Z$ stand for arbitrary but fixed (crisp) sets, the small letters $f$, g stand for arbitrary but fixed (crisp) maps $f: X \rightarrow Y$ and $g: Y \rightarrow Z$, the capital letters A, B, C, D, E, F together with their suffixes stand for if/V-subsets and the capital letters I and J stand for the index sets. In general whenever $\mathrm{P}$ is an if/v-subset of a set $\mathrm{X}$, always $\mu_{\mathrm{P}}$ and $\mathrm{u}_{\mathrm{P}}$ denote the membership and non membership function of the if $/ \mathrm{v}$ subset $\mathrm{P}$ respectively. Also we frequently use the standard convention that $\mathrm{V} \Phi=0$ and $\Lambda \Phi=1$.

\section{Main Results}

In this section the notions and properties of Intuitionistic fuzzy/vague image and Intuitionistic fuzzy/ vague inverse image for an Intuitionistic fuzzy/vague subset of a set under a crisp map are recalled and are shown to be well defined.

\section{1 if-Images and if-Inverse Images}

Let $\mathrm{X}, \mathrm{Y}$ be a pair of sets and let $\mathrm{f}: \mathrm{X} \rightarrow \mathrm{Y}$ be a map. Let $\mathrm{A}=\left(\mu \mathrm{A}, v_{\mathrm{A}}\right)$ and $\mathrm{B}=(\mu \mathrm{B}, v \mathrm{~B})$ be if $/ \mathrm{v}$ subsets of $\mathrm{X}$ and $\mathrm{Y}$ respectively. 
Definitions and Statements 2.1.1: (1) Let $\mu_{D}, \nu_{D}: Y \rightarrow[0,1]$ be defined by $\mu_{D} y=\mu_{A} f^{-1} y$ and $v_{D} y=\Lambda v_{A} f^{-1} y \forall y \in Y$. Then $D$ is a well defined if $/ v$-subset of $Y$ as follows:

Let $\mathrm{y} \in \mathrm{Y}$. It is enough to show that $\mu_{D} \mathrm{y}+\mathrm{v}_{\mathrm{D}} \mathrm{y} \leq 1$. If $\mathrm{f}^{-1} \mathrm{y}$ is empty, we are done because $\mathrm{V} \Phi=0$ and $\Lambda \Phi=1$. Therefore, let $\mathrm{f}^{-1} \mathrm{y} \neq \Phi$. Then $\mu_{\mathrm{A}} \mathrm{x} \leq 1-\mathrm{v}_{\mathrm{A}} \mathrm{x} \leq 1-\Lambda \mathrm{v}_{\mathrm{A}} \mathrm{f}^{-1} \mathrm{y}$ for all $\mathrm{x}$ in $f^{-1} y$ which implies $V_{x \in f-1 y} \mu_{A} x \leq 1-\Lambda v_{A} f^{-1} y$ or $V \mu_{A} f^{-1} y+\Lambda v_{A} f^{-1} y \leq 1$.

(2) Let X, Y, f and A be as above. Then the if $/ v$-subset D of Y defined as in (1) above, called the Intuitionistic fuzzy/vague image of A under f or simply the If/v-image of A under $\mathrm{f}$, is denoted by fA.

(3) Let $\mu_{C}, v_{C}: Y \rightarrow[0,1]$ be defined by $\mu_{C} x=\mu_{B} f x$ and $v_{C} x=v_{B} f x, \forall x \in X$. Then $C$ is a well defined if $/ \mathrm{V}$-subset of $\mathrm{X}$ as follows:

Let $\mathrm{x} \in \mathrm{X}$. It is enough to show that $\mu_{C} \mathrm{X}+\mathrm{v}_{\mathrm{C}} \leq 1$ or $\mu_{\mathrm{B}} f \mathrm{x}+\mathrm{v}_{\mathrm{B}} \mathrm{fx} \leq 1$ which is always there because $\mathrm{B}$ is a well defined if $/ \mathrm{v}$-subset of $\mathrm{Y}$.

(4) Let X, Y, f and B be as above. Then the if/v-subset C of X defined as in (3) above, called the Intuitionistic fuzzy/vague inverse image of B under $f$ or simply the if $/ v$-inverse image of $B$ under $f$, is denoted by $\mathrm{f}^{-1} \mathrm{~A}$.

\subsection{Mapping Properties of if-Images and if-Inverse Images}

In this section we show that several of the mapping properties that hold good for Zadeh fuzzy subsets are also held good for the Intuitionistic fuzzy subsets.

2.2.1 Theorem Let $X, Y$ be a pair of sets and let $\mathrm{f}: \mathrm{X} \rightarrow \mathrm{Y}$ be a map. Let $\mathrm{A}, \mathrm{Ai}$ and $\mathrm{B}, \mathrm{B}_{\mathrm{i}}$ be if-subsets of $\mathrm{X}$ and $\mathrm{Y}$ respectively. Then the following are true:

1. $\mathrm{A} \leq \mathrm{C}$ implies $\mathrm{f} \mathrm{A} \leq \mathrm{fC}$

2. $B \leq D$ implies $f^{-1} B \leq f^{-1} D$

3. always $A \leq f^{-1} f A$

4. $A=f^{-1} f A \Leftrightarrow f$ is $1-1$

5. always $\mathrm{ff}^{-1} \mathrm{~B} \leq \mathrm{B}$

6. $\mathrm{ff}^{-1} \mathrm{~B}=\mathrm{B} \Leftrightarrow$ if $\mathrm{f}$ is onto

7. $f\left(V_{i \in 1} A_{i}\right)=V_{i \in 1} f A_{i}$

8. $f\left(\Lambda_{i \in 1} A_{i}\right) \leq \Lambda_{i \in 1} f A_{i}$ and the equality is true whenever $f$ is $1-1$.

9. $\mathrm{f}^{-1}\left(\mathrm{~V}_{\mathrm{i} \in 1} \mathrm{~B}_{\mathrm{i}}\right)=\mathrm{V}_{\mathrm{i} \in 1} \mathrm{f}^{-1} \mathrm{~B}_{\mathrm{i}}$

10. $\mathrm{f}^{-1}\left(\Lambda_{\mathrm{i} \in 1} \mathrm{~B}_{\mathrm{i}}\right)=\Lambda_{\mathrm{i} \in 1} \mathrm{f}^{-1} \mathrm{~B}_{\mathrm{i}}$

11. $f A=\varphi \Leftrightarrow A=\varphi$, in particular, $f \varphi=\varphi$, and when $f$ is $1-1, f A=f X \Leftrightarrow A=X$

12. $\mathrm{f}^{-1} \mathrm{~B}=\mathrm{X} \Leftrightarrow \mathrm{B} \geq \mathrm{fX}$, in particular, $\mathrm{f}^{-1} \mathrm{fX}=\mathrm{X}$ and $\mathrm{f}^{-1} \mathrm{~B}=\varphi \Leftrightarrow \mathrm{B} \leq \mathrm{Y}-\mathrm{fX}$, in particular, $\mathrm{f}^{-1} \varphi=\varphi$

13. $f X-f A \leq f(X-A)$ and the equality holds whenever $f$ is $1-1$

14. $f^{-1}(Y-B)=X-\left(f^{-1} B\right)$

15. $\mathrm{ff}^{-1} \mathrm{~B}=\mathrm{B} \Lambda \mathrm{fX}$ and hence always $\mathrm{ff}^{-1} \mathrm{~B} \leq \mathrm{B}$

16. $\mathrm{f}^{-1} \mathrm{~B}=\mathrm{f}^{-1}(\mathrm{~B} \wedge \mathrm{fX})$

17. $\mathrm{fA} \leq \mathrm{B} \Leftrightarrow \mathrm{A} \leq \mathrm{f}^{-1} \mathrm{~B}$

18. (i) $\mathrm{ff}^{-1} \mathrm{fA}=\mathrm{fA}$ (ii) $\mathrm{f}^{-1} \mathrm{ff}^{-1} \mathrm{~B}=\mathrm{f}^{-1} \mathrm{~B}$

2.2.2 Theorem Let $X, Y$ and $Z$ be three sets and let $f: X \rightarrow Y$ and $g: Y \rightarrow Z$ be a pair of maps. Then the following are true:

1. $(g \circ f)(A)=g(f(A))$ for all $A \leq X$

2. $(\mathrm{g} \circ \mathrm{f})^{-1}(\mathrm{C})=\mathrm{f}^{-1}(\mathrm{~g}-\mathrm{C})$ for all $\mathrm{C} \leq \mathrm{Z}$

\section{Acknowledgements:}

The author would like to express her heart full thanks to the Dr.N.V.E.S.Murthy, Department of Computer Science and System Engineering, AUCE, Andhra University, for his constant 
encouragement and help throughout the preparation of this paper. The author would like to express her thankfulness to the management of Aditya Institute of Technology and Management, Tekkali for their inspiration and support.

\section{References}

[1] Atanassov, K., Intuitionistic Fuzzy Sets, V. Sgurev, Ed., VII ITKR's Session, Sofia, June 1983, Bulgarian Academy of Sciences, 1984).

[2] Atanassov, K. and Stoeva, S., Intuitionistic Fuzzy Sets, Polish Symp. On Interval and Fuzzy Mathematics, Poznan, August 1983, P23-26.

[3] Atanassov, K. and Stoeva, S., Intuitionistic L-fuzzy Sets, R. Trappl, Ed., Cybernetics and Systems Research 2, Elsevier Science Publishers, Amsterdam, 1984, P539-540.

[4] Davvaz B., Dudek W.A. and Jun Y.B, Intuitionistic fuzzy Hv - submodules, Information Sciences, Vol.176(3)(2006), P285-300.

[5] Galindo, J., Urrutia, A. and Piattini, M., Fuzzy databases: Modeling, Design and Implementation, Idea Group Publishing, London, 2006.

[6] Gau, W.L. and Buehrer D.J., Vague Sets, IEEE Transactions on Systems, Man and Cybernetics, Vol. 23(1993), P610-614.

[7] Hiroshi S., Rough Sets, Fuzzy Sets, Data Mining and Granular Computing: 12th International Conference,RSFDGrC 2009, Delhi, India, Proceedings, Springer, 2009, ISBN 3642106455.

[8] Jose Valente de Oliveira and Witold Pedrycz, Advances in fuzzy clustering and its applications, John Wiley and Sons Inc. New York, USA, 2007 ISBN: 0470027606.

[9] Morgan K., Fuzzy Modeling and Genetic Algorithms for Data Mining and Exploration, 1st edition (2005), ISBN-10: 012-1942759, ISBN-13:978-0121942755.

[10] Mordeson J.N. and Malik D.S., Fuzzy Commutative Algebra, World Scientific, Singapore, 1998.

[11]Puyin Liu, Hong-Xing Li, Fuzzy Neural Network Theory and Application, World Scientific, 2004.

[12] Tamalika C. and Ajoy Kumar Ray, Fuzzy Image Processing and Applications with MATLAB, CRC Press; 1st edition, November 24, 2009, ISBN-10: 1439807086, ISBN-13: 9781439807088.

[13]Thakur.S.S and Jyoti Pandey Bajpai, Intuitionistic Fuzzy W-Closed Sets and Intuitionistic Fuzzy W - Continuity, International Journal of Contemporary Advanced Mathematics (IJCM), Volume (1): Issue (1).

[14] Yan-Qing Zhang, Compensatory Genetic Fuzzy Neural Networks and Their Applications, (Series in Machine Perception and Artificial Intelligence), World Scientific Pub Co Inc, November 1998.

[15] Ying-Ming and Mao-Kang, Fuzzy Topology, World Scientific, Singapore, 1997.

[16] Yong Ho Yon, Young Bae Jun and Kyung Ho Kim, Intuitionistic Fuzzy R-Subgroups Of Near Rings, Soochow Journal Of Mathematics, Vol.27(2001), no.3, P243-253.

[17]Jian-Ming, Interval Valued Intuitionistic Fuzzy Hv-Subgroups, Journal of Mathematical Research and Exposition 2007-04. 